\title{
Was ist ein Franz-Ferdinand-Roman? \\ Und warum schreibt man einen solchen 1937? \\ Zu Ludwig Winders Der Thronfolger
}

\section{What is a Franz Ferdinand novel? And why writing such \\ a novel in 1937?}

About The heir to the throne by Ludwig Winder

Manfred Weinberg

\begin{abstract}
The article describes Ludwig Winder's novel The heir to the throne. A Franz Ferdinand novel as both a psychological study and a historical novel. But as Franz Ferdinand never became emperor (and king), the novel is also an ,alternative history'. From the perspective of the ruler to be the other possibilities of the state of the pluricultural Habsburg monarchy and Europe are reflected, which are still relevant for the present of Winder, which was overshadowed by the rise of Adolf Hitler, and also for our own present. This also explains why Winder wrote his Franz Ferdinand novel 23 years after the assassination of the heir to the throne in Sarajevo.
\end{abstract}

\section{Keywords}

Ludwig Winder; The heir to the throne; Franz Ferdinand; Habsburg monarchy; historiography; historical novel; alternative history 
Im Frühjahr 1911 ging Ludwig Winder nach Teplitz und wurde Redakteur der deutschliberalen Teplitzer Zeitung. In Kurt Krolops Nachwort zur Ausgabe des Thronfolger-Romans von 1984 liest man:

„Es ist gewiß kein Zufall, daß der einzige Leitartikel, den Winder neben Schauspiel-, Opernund Operettenbesprechungen, Gedichten und Feuilletons für die Teplitzer Zeitung schrieb, ,Franz Ferdinand“ überschrieben ist.

Das Rätsel, das die düstere Persönlichkeit des Thronfolgers den Zeitgenossen aufgab, scheint Winder schon damals beschäftigt zu haben, und manches in dem Leitartikel klingt, als stelle der Autor sich hier selbst eine Aufgabe:

,Für uns Österreicher ist es unendlich schwer, einer Persönlichkeit gegenüber die richtige Distanz zu gewinnen, die bisher immer nur hinter verschlossenen Türen wirkte und von der eigentlich nichts weiter mit Bestimmtheit behauptet werden kann, als daß sie den Anfang einer neuen österreichischen Epoche zu bilden bestimmt ist. Das österreichische Antlitz Franz Ferdinands ist undurchdringlich [...]. Die Psychologie des Thronfolgers ist nun gar ein Kapitel, das erst darauf wartet, aufgeschlagen zu werden.“ (Krolop 1984: 605)

Kurt Krolop kommentiert: „Damit hatte Winder zum ersten Male ein Problem berührt, auf das er als Journalist immer wieder zurückkam, bis er es schließlich in seinem Franz-Ferdinand-Roman dichterisch bewältigt hatte.“ (Krolop 1984: 605)

Von 1911 aus gesehen ist der Thronfolger Franz Ferdinand die vermutlich nahe Zukunft; doch sein ,Antlitz‘ und damit auch das Gesicht und Gepräge der Zukunft sind undurchdringlich, seine Psychologie ein noch nicht aufgeschlagenes Kapitel. Im Begriff „Kapitel“ kann man, wenn man will, den Hinweis darauf finden, dass eben die Psychologie des Thronfolgers Gegenstand eines Romans werden sollte, um sie in aller Breite zu erkunden. Warum aber wird dieser Roman erst 1937 geschrieben, als der Thronfolger schon 23 Jahre tot ist, als es die k.u.k-Monarchie Österreich-Ungarn nicht mehr gibt und als sich abzeichnet, dass noch ganz andere Veränderungen den europäischen Kontinent erschüttern werden, schließlich war Adolf Hitler schon vier Jahre an der Macht? Eine Befassung mit Franz Ferdinand zu diesem Zeitpunkt war eine Befassung mit der Vergangenheit; über die Zukunft konnte dieses Projekt nun keine Auskunft mehr geben. Oder ist das zu einfach gedacht, weil es davon ausgeht, dass Der Thronfolger ein historischer Roman ist? Zu diesem Genre heißt es im entsprechenden Kapitel des Handbuchs der deutschen Literatur Prags und der Böhmischen Länder, dass es „als stringente, einheitliche Gattung kaum auszumachen“ (Höhne - Mayer - Weinberg 2017: 358) sei. Zitiert wird dann allerdings die Einschätzung Harro Müllers aus seiner Studie Geschichte zwischen Kairos und Katastrophe. Historische Romane im 20. Jahrhundert, dass der historische Roman der „Dominanz personell-individueller Triebkräfte in der Geschichte“ verpflichtet sei und auf Präsuppositionen der Kausalität, der Narrativität, der Diachronie historischer Abläufe basiere, durch die ein per „Sprache mimetisch“ abbildbares, „universal-geschichtliches Kontinuum“ (Müller 1988: 17-18) entstehe. Auf Winders Thronfolger scheint mir das allerdings nicht zuzutreffen. Von der „Dominanz personell-individueller Triebkräfte“ handelt der Roman deshalb nicht, weil Franz Ferdinand, worauf zurückzukommen sein 
wird, als Herrscher im ,Wartestand' ja kaum in die Geschichte eingreifen konnte. Und für ein „universal-geschichtliches Kontinuum“ ist der Roman dann eben doch viel zu sehr auf seinen Protagonisten fixiert, woran ich ebenfalls unten noch einmal anschlieBen werde.

In den Rezensionen zur Neuausgabe von 2014 gab es sehr verschiedene Aussagen dazu, welcher Gattung der Roman zuzurechnen ist. Da ist von einem „biografischen Roman“ (Leitner 2014) oder einer „Romanbiografie“ (Seibt 2014) die Rede sowie von einer „psychologische[n] Studie“ (Henneberg 2014). Ein gewisser Andreas schreibt im LiteraturBlog von LeserInnen für LeserInnen, der Roman sei „vieles in einem: ein Gesellschaftsroman und eine Biographie, ein Entwicklungsroman und ein historischer Roman" (Andreas 2014). F.C. Weiskopf, einer der ersten Rezensenten der Erstausgabe, hat ihn im März-Heft der Moskauer Exil-Zeitschrift Das Wort 1938 ein „bürgerliches Trauerspiel“ (zit. nach Krolop 1984: 624) genannt. Dazu passt die Einschätzung aus dem Handbuch der deutschen Literatur Prags und der Böhmischen Länder: „Der Thronfolger ist weder ein Tatsachenroman noch ein [wie Kurt Krolop dementiert hat; M.W.] ,historisches Kolossalgemälde zum Thema ,Finis Austriae“ [...] und auch keine Biographie, sondern eher ein Familiendrama“" (Höhne - Mayer - Weinberg 2017: 364). Walther Victor hat in seiner Besprechung vom November 1937 im Escher Tagblatt geschrieben, der Roman wolle „das Ende als Ausgang im Sinne der klassischen Tragödie darstellen, an deren Ende das Eingreifen einer überirdischen Schicksalsmacht den dramatischen Schlußpunkt setzt“ (Victor 1937). Wie Victor auf die „überirdische Schicksalsmacht" kommt, hat sich mir nicht erschlossen. Interessant ist aber allemal, dass ich soeben drei Einschätzungen zitieren konnte, die den Roman als ,bürgerliches Trauerspiel', ,Familiendrama' und ,Tragödie‘ strukturell mit einem Drama vergleichen, was mit dem hohen Stellenwert der ,Figurenrede“ in diesem Roman zu tun hat. Das äußert sich über ausgiebig zitierte mündliche Rede hinaus oft auch in der Fokalisierung auf eine Figur, deren Blick auf die Welt in erlebter Rede kolportiert wird und dies nicht nur bezüglich Franz Ferdinands.

Im Weiteren werde ich mich allerdings nicht mehr mit der Rezeption dieses Romans befassen, denn es gab kaum eine, jedenfalls keine deutschsprachige: Der 1937 im Zürcher Humanitas-Verlag veröffentlichte Roman durfte in Deutschland nicht verkauft werden, weil der Verfasser Jude war, in Österreich wurde er auf der Grundlage des Traditionsschutz-Gesetzes von 1935 verboten. Eine tschechische Übersetzung von František Šelepa erschien im April 1938 in der Reihe Knihovna Lidových novin; die daran anschlieBende tschechische Rezeption behandelt in diesem Heft Ladislav Futtera. 1984 kam der Roman mit einem Nachwort von Kurt Krolop noch einmal in der DDR heraus. Marcel Reich-Ranicki ließ ihn 1990 im dritten Band seiner Reihe Romane von gestern - heute gelesen besprechen. Schließlich erschien 2014 die Neuausgabe im Zsolnay-Verlag, zu der Thomas Leitner im österreichischen Falter schrieb, dass es sich „[f]ür den größten Teil des deutschsprachigen Publikums [...] um eine Erstbegegnung“" (Leitner 2014) handle. Die Besprechungen waren durchweg äußerst positiv. Immer wieder wurde Winders Thronfolger über Joseph Roths Radetzkymarsch gestellt (vgl. z.B. Leitner 2014).

Zurück aber zur Gattung. Worum handelt es sich denn nun: einen biographischen Roman, eine Romanbiographie, was durchaus einen Unterschied macht, einen 
Gesellschaftsroman, einen Entwicklungsroman oder einen historischen Roman? Andreas (Nachname unbekannt) vom LiteraturBlog hat schon recht - dieser Roman ist alles zugleich, wobei sich in die Zurechnungen ja durchaus Ordnung bringen lässt: Gesellschafts- und historischer Roman zielen aufs Große und Ganze, Biographie und Entwicklungsroman sowie „psychologische Studie“ auf einen Einzelnen. Im Beitrag zum Ersten Weltkrieg im Handbuch der deutschen Literatur Prags und der Böhmischen Länder heißt es über Winders Roman in eben diesem Sinne: Winders Thronfolger

„trägt im Gegensatz zu Brehms Erzherzog [in dessen Franz-Ferdinand-Roman Apis und Este von 1931; M.W.] fast ausschließlich negative bis pathologische Züge. Diese werden auf die Vorfahren des Erzherzogs und somit auf die Eliten des alten Europa zurückgeführt, wobei Franz Ferdinand als Verkörperung ihrer negativen Eigenschaften gezeichnet und als potenzieller Tyrann dargestellt wird. Die ,Abrechnung' mit dem Thronfolger wird gleichzeitig zur Abrechnung mit den Eliten der Doppelmonarchie und des Deutschen Kaiserreichs, denen der auktoriale Erzähler die Schuld am Ausbruch des Weltkriegs zuschreibt.“ (Horňáček - Schöning 2017: 341)

Schon in Winders Auseinandersetzung mit Franz Ferdinand in der Teplitzer Zeitung ist eine solche ,Zweiseitigkeit' zu erkennen: Da geht es einmal um das undurchdringliche Antlitz und die Psychologie des Thronfolgers - also um ihn als Individuum -, andererseits um ihn als denjenigen, der „eine neue österreichische Epoche zu bilden bestimmt ist“, also ums Große und Ganze. Margarita Pazi beschrieb diese ,Zweiseitigkeit“ als Winders Ziel, „in exaktem historischem Rahmen einen Seelenroman zu entwickeln“ (Pazi 2001: 111). Damit leistet der Roman aber etwas, was einer anderen journalistischen Beschäftigung Winders mit Franz Ferdinand - einem Artikel in der Vossischen Zeitung vom Oktober 1930 unter dem Titel Das Heim eines finsteren Menschen, der von einem Besuch in Konopischt, dem langjährigen Wohnsitz Franz Ferdinands, handelt - fehlt, denn dieser ist mehr eine Abrechnung mit dem Thronfolger als ein komplexes Psychogramm. Er war, schreibt Winder darin, „ein gieriger, fanatischer, unersättlicher Mensch“. Und: „Noch heute glüht in den Augen der älteren Bewohner von Beneschau der Hass, wenn sie von ihm sprechen. [...] Vielleicht haben sie gewusst, dass er unglücklich war. Sein Unglück milderte nicht ihren Hass." (Winder 1930) Ulrich Weinzierl führt diese Diagnose in seinem Nachwort zur Ausgabe von 2014 übrigens „auf die Sicht des verkürzenden, zuspitzenden Tagesschreibers“ zurück, die der Romancier zugunsten eines „vollplastische[n] Bildes“ (Weinzierl 2014: 556) überwunden habe. Das scheint mir allerdings nur dann angemessen, wenn man sich klar macht, dass der Roman eben mehr sein wollte als ein bloßes Psychogramm, nämlich auch ein historischer (und aktueller) Roman, was nur gelingen konnte, wenn er nicht das Porträt eines Thronfolgers als Bösewicht bot, sondern die ganze Ambivalenz jeder historischen Situation auch in der Auseinandersetzung mit dem Innenleben des Thronfolgers erwies. Heinz Politzer hat Ende November 1937 in seiner Besprechung des Thronfolgers im Prager Tagblatt von Winder als einem "durchgebildete[n] Kenner moderner Seelenkunde“ geschrieben, der das Leben Franz Ferdinands als das „eines nie völlig erlösten Menschen“ (Politzer 1937) beschreibe. Dass dies gelungen ist, hat meiner Ansicht nach aber damit zu tun, das Winder im Roman 
eben immer auch das nie völlig Erlöste Österreich-Ungarns und Europas im Blick hat.

Bei der Reflektion der Frage, welcher Gattung der Thronfolger zuzurechnen ist, sind die Rezensenten fast nie auf die Gattungsbezeichnung eingegangen, die Winder seinem Roman selbst gegeben hat: Ein Franz-Ferdinand-Roman. Was aber ist ein Franz-Ferdinand-Roman? Aristoteles hat Gattung folgendermaßen definiert: „Gattung ist, was von mehreren und der Art nach verschiedenen Dingen bei der Angabe ihres Was oder Wesens prädiziert wird." (Aristoteles 1995: I,5, 102a31). Verschiedenes muss also gewisse Prädikate gemeinsam haben, um zu einer Gattung gerechnet werden zu können. In Wikipedia - ich weiß: ein großer Schritt - liest man zu literarischen Gattungen:

„Die Gattungsbestimmung eines Textes geschieht in der Weise, dass typische formale Aspekte eines Überlieferungsstücks mit anderen verglichen werden [...]. Ergeben sich Übereinstimmungen, so darf angenommen werden, dass die verglichenen Stücke der gleichen Gattung angehören." (Wikipedia 2020, mit Verweis auf Lehnardt 2002: 64)

Als Roman kommt ein Franz-Ferdinand-Roman also mit anderen in vielem überein; als Franz-Ferdinand-Roman ist er jedoch unvergleichlich, weil es einen solchen eben nicht noch einmal gibt. Bei näherer Betrachtung erweist sich der Titel allerdings als noch komplexer. Im Haupttitel ist vom Thronfolger die Rede, von dem im Übrigen gilt, was ich eben zur Gattungsangabe Franz-Ferdinand-Roman gesagt habe: Es kann für ein Herrscherhaus auch immer nur einen Thronfolger geben. Der Nennung des Namens im Untertitel geht also im Haupttitel sozusagen seine Funktionsbestimmung voraus, diese allerdings versehen mit dem bestimmten Artikel: Gemeint ist dieser eine Thronfolger, zunächst in seiner Funktion, dann mit seinem Namen bestimmt. Dass beides getrennt wird, scheint mir die eben schon diagnostizierte Spannung und ,Zweiseitigkeit' zu bestätigen. Es ist ein Roman, der zugleich vom Einzelnen und vom Großen und Ganzen handelt. In den beiden wohl beeindruckendsten Szenen des Romans lässt sich diese Konstellation wiederfinden: in der Szene der Renunziation (also Franz Ferdinands Unterschrift unter das Dokument, das seine Ehe mit der Gräfin Chotek zu einer morganatischen Ehe oder Ehe linker Hand erklärt und ihre gemeinsamen Kinder von der Erbfolge ausschließt) und in der Erzählung vom „Hofbegräbnis Dritter Klasse“ (Winder 1984: 588, 590), bei dem die Särge „, [z]wischen den Geleisen, im rauschenden Regen, im heulenden Sturm, von Blitzen umzuckt, [...] länger als eine Stunde liegen“ (Winder 1984: 590-591). Zur Renunziation heißt es:

\footnotetext{
„Der Kaiser betrat den Korridor, die Erzherzoge, die in dem großen Audienzsaal seine Ankunft abgewartet hatten, schlossen sich ihm an. Er salutierte, nickte ihnen zu, blickte keinen an. Er blickte keinen Menschen an, als er die Geheime Ratsstube betrat. Er ging langsam auf den unter einem prunkvollen Baldachin stehenden Thronsessel zu und setzte sich. Der Erste Obersthofmeister ging auf Franz Ferdinand zu und bat ihn, rechts vom Thronsessel auf der Estrade stehen zu bleiben. Links vom Thronsessel nahmen alle andern Erzherzoge Aufstellung. In der Mitte stand der Minister des Auswärtigen und des Kaiserlichen Hauses, Graf Goluchowski, in kleinem Abstand der Kardinal-Fürsterzbischof von Wien. Vor der Front der
} 
Erzherzoge reckte sich die zierliche schmächtige Gestalt des Zweiten Obersthofmeisters, Fürsten Montenuovo. Unterhalb der Estrade standen, dicht gedrängt, die übrigen Geladenen: die Generale, die Bischöfe, die Minister, die Geheimen Räte, die Kämmerer, die hohen Beamten. Wie viele Feinde! dachte Franz Ferdinand.“ (Winder 1984: 357-358)

Hier stehen dem Thronfolger als Einzelnem also die versammelten Vertreter des Staates gegenüber.

Beim Begräbnis fehlen dann alle Staatsoberhäupter, die ihr Kommen angekündigt hatten, weil Graf Montenuovo ihnen mitgeteilt hatte, dass „auf das übliche Zeremoniell wegen der vorliegenden Umstände verzichtet werde“, so dass den „Staatsoberhäuptern auch nicht zugemutet werden könne, zu der in aller Stille stattfindenden Totenfeier einen Vertreter zu entsenden“ (Winder 1984: 587). Zudem untersagt er „allen Geheimen Räten und allen Kämmerern, an dem Leichenbegängnis teilzunehmen“ (Winder 1984: 589). Anwesend sind dann als sozusagen ,mindere“ Vertreter des Staates „alle Veteranenund Feuerwehrvereine aus Pöchlarn und den umliegenden Orten“ und die „Angestellten der Wiener städtischen Leichenbestattung“ (Winder 1984: 590). Dass das Fehlen der Staatsoberhäupter, Geheimen Räte und Kämmerer jedoch so ausführlich beschrieben wird wie die Anwesenheit nur untergeordneter Staatsvertreter, gibt der Begräbnisszene zuletzt die gleiche Struktur wie sie die Zeremonie der Renunziation hat. Winder entscheidet sich nicht zwischen Psychogramm und historischem Roman - er stellt vielmehr die Frage nach dem Zusammenhang von Einzelnem und Geschichte (und das nicht nur bezüglich der Zentralfigur Franz Ferdinand). Von daher ist es aber anderes und mehr als ein Roman über ,Kakanien“ - oder mit den Worten Kurt Krolops: Dieser Roman steht nur scheinbar im Kontext einer „schwarz-gelben Nostalgiewelle“, da der Protagonist „das genaue Gegenteil jener Tugenden oder Untugenden der Konnivenz, Konzilianz und Kulanz verkörperte“ (Krolop 1984: 621), aus denen sich der ,habsburgische Mythos zusammensetzte.

So ist der Roman keinesfalls ein nostalgischer Rückblick auf die k.u.k.-Monarchie, was aber zur im Titel meines Beitrags gestellten Frage führt, warum sonst man einen Roman über den Thronfolger Franz Ferdinand erst 1937, 23 Jahre nach dem tödlichen Attentat auf ihn, schreibt? Dabei ist diese Frage für mich auch sozusagen in die andere Richtung gültig: Es scheint mir durchaus naheliegend, dass man aus dem noch weiteren Abstand des Jahres 1961 - also mitten im Kalten Krieg - auf Franz Ferdinand zurückkommt, wie das der österreichische Schriftsteller Ernst Joseph Görlich mit seinem Roman Der Thronfolger getan hat, in dem Franz Ferdinand als Hoffnung und möglicher Retter der habsburgischen Monarchie erscheint. Warum aber 1937? Schon die erwähnte Besprechung der Erstausgabe durch Walther Victor im Escher Tagblatt beginnt mit dem Hinweis, dass man sich über das „späte Erscheinen“ des Buches wundere, doch mute es „in unseren Tagen unerhört aktuell“ (Victor 1937) an.

Auch die Forschung hat die Frage nach dem Gegenwartsbezug des Romans schon gestellt. So schreibt Christian Jäger in seiner Studie Minoritäre Literatur. Das Konzept der kleinen Literatur am Beispiel prager- und sudetendeutscher Werke über den Thronfolger, den er einseitig als historischen Roman versteht, im Verweis auf Krolops Winder-Zitat aus der 
Teplitzer Zeitung, mit dem ich diesen Aufsatz begonnen habe:

„Die nahegelegte Kontinuität [zwischen Leitartikel und Roman; M.W.] erklärt jedoch weniger, als dass sie das erklärungsbedürftige Problem aufwirft, warum Winder denn nun nach über zwei Dekaden den Roman geschaffen hat, der die so früh gestellte Aufgabe annimmt und zu bewältigen sucht." (Jäger 2005: 432)

Jägers Antwort liegt darin, den Roman für eine verkappte Auseinandersetzung mit dem Faschismus zu halten. Ich zitiere ausführlicher: Eine „Nähe zu faschistischem Denken“ werde kenntlich,

„wenn Franz Ferdinand seine Sympathie für den antisemitischen Demagogen, anti-magyarischen Populisten und christsozialen Bürgermeister Wiens, Karl Lueger, bekundet. Die Aktualität des ,historischen' Romans wird ebenso evident wie das geringe Ausmaß, in dem Winder mit diesem Buch nur eine persönliche und zudem historische Aufgabe bewältigt. Betrachtet man den Roman unter diesem Aspekt als Auseinandersetzung mit dem faschistischen Herrschaftsprinzip, so geht es in ihm nicht allein um die Psychologie des Führers, sondern auch in einem recht unmittelbaren Sinn um politische Praxis.“ (Jäger 2005: 435)

Jäger geht noch einen Schritt weiter, wenn er formuliert:

„Die [durch das Ende von Winders Roman; M.W.] implizit gestellte Frage, macht es Sinn, Hitler umzubringen, erfährt also eine bejahende Antwort, verknüpft sich mit der Hoffnung, dass auf diese Weise der Faschismus überwunden werden könne, und zugleich mit der Warnung, dass der Weg zur Katharsis über die Katastrophe führt und führen kann.“ (Jäger 2005: 438)

Bisher war ich der Meinung, dass erst in jüngerer Zeit vergleichsweise wahllos das Etikett ,Faschist" vergeben wird, doch in Jägers Studie von 2005 findet es sich umstandslos auf den Wiener Bürgermeister Karl Lueger angewendet. Dieser war sicher ein übler Antisemit - aber wirklich auch ein Faschist, was die Anbindung des Winderschen Romans an seine Entstehungszeit erklären soll? Zudem allgemein: Der Thronfolger als Roman über den Faschismus und das Führerprinzip? Diese Diagnose geht schon deshalb nicht auf, weil Franz Ferdinand niemals zum Führer wird und sozusagen als ,Herrscher im Wartestand' in Sarajevo ermordet wird.

Von daher sehe ich eine ganz andere Anbindung an die Gegenwart der Textentstehung. Gerade weil der Thronfolger nicht Kaiser (und König) ist (und niemals wird), sind alle seine Pläne nur Möglichkeiten: von den „Vereinigten Staaten von Österreich-Ungarn“ (Winder 1984: 211) (so wörtlich im Roman) bis hin zum „Trialismus“ (Winder 1984: $296 \mathrm{u}$. ö.), also dem Plan, „[d]as Reich in drei autonome Gebiete zu teilen: ein österreichisches, ein ungarisches und ein südslawisches“ (Winder 1984: 296). Die ausgiebig dargelegten Pläne erschaffen alternative Wirklichkeiten und sind eben keine historische Realität. Als bloße Möglichkeiten gehen sie aber eben auch über die zeitgenössische Wirklichkeit Franz Ferdinands hinaus und entwerfen eine ,alternative history oder - wie dieses Gerne in 
der Geschichtsschreibung heißt - eine ,kontrafaktische Geschichte'. Nach Nicole Hennebergs Rezension in der Frankfurter Allgemeinen Zeitung eröffnet das Buch „den Blick auf die Utopie eines freiheitlichen Europa“, aber auch ihr „Scheitern“ (Henneberg 2014).

Natürlich schreibt Winder eine ,alternative history" nicht in der radikalen Weise wie Hannes Stein in seinem Roman Der Komet von 2013, der davon ausgeht, dass Franz Ferdinand, nach dem ersten, gescheiterten Versuch, ihn in Sarajewo umzubringen, den vernünftigen Entschluss fasst: „I bin do ned deppat, i fohr wieder z'Haus.“ (zitiert nach Gauß 2014). Da es somit keinen ermordeten Thronfolger gibt, kommt es zu keinem Ultimatum an Serbien, nicht zum Ersten und, daraus resultierend, auch nicht zum Zweiten Weltkrieg. Die europäischen Staaten versuchen, ihre komplizierte Machtbalance zu erhalten. Amerika ist ein zurückgebliebener Kontinent voller Cowboys, Goldgräber und Hinterwäldler; Europa bleibt das vorherrschend von Monarchen regierte Maß aller Dinge. Vor allem: Das k.u.k.-Reich mit seiner Hauptstadt Wien ist und bleibt der Nabel der Welt (vgl. Gauß 2014). Das ist eine hübsche Kontrafaktur der Geschichte, aber extrem weit von Winders Thronfolger entfernt, der sich in seinem einzigen historischen Buch so genau an gründlichst recherchierte Fakten hält, dass Ulrich Weinzierl in seinem Nachwort zur Neuausgabe von 2014 darauf hinweisen kann, dass der Roman „mit zureichendem Grund“ in den „Bibliographien der bekanntesten historischen Sachbücher“ (Weinzierl 2014: 560) über Franz Ferdinand auftaucht. Das aber macht das Phänomen ja nur noch interessanter. Aus dem Status des Noch-Nicht-Herrschers werden die anderen Möglichkeiten des Zustands Kakaniens und Europas reflektiert, was sie auch noch für die Gegenwart Winders relevant sein lässt.

Als Franz Ferdinand gerade zum Thronfolger ernannt worden ist, denkt er: „Aber ich fühl, so schrecklich klar fühl ich das schon längst, dass etwas in mir steckt, etwas, wovon niemand eine Ahnung hat, etwas, das mich groß machen wird, mich und das Reich, wenn mir Gott die Gelegenheit geben wird, zu zeigen, was in mir steckt." (Winder 1984: 143) Die Gelegenheit aber wird er nicht bekommen, so dass seine Pläne eben immer nur im Zustand des Möglichen, des „Ich will!“ (so in langer Reihe ausformuliert am Ende des Kapitels Thronfolger, vgl. Winder 1984: 161) verbleiben. Einmal heißt es: „Man musste den Nationen beibringen, dass die vielen kleinen Staaten, in die Österreich-Ungarn zerfiele, wenn es das große Reich nicht mehr gäbe, der Willkür aller expansionistischen Großmächte ausgesetzt wären.“ (Winder 1984: 213) An anderer Stelle:

„Allen Nationen musste beigebracht werden, dass das Habsburgerreich eine europäische Notwendigkeit war und dass sie Selbstmord begingen, wenn sie die Einheit, den Bestand der Monarchie untergraben wollten. Aber wie konnte diese Idee gefestigt werden?“ (Winder 1984: 205)

Thomas Leitner hat im Falter geschrieben:

„Nicht um Abrechnung mit dem Vielvölkerstaat, einer vergangenen, unmöglich gewordenen Notwendigkeit, geht es Winder, nicht um einen Nachruf wie bei Joseph Roth, sondern um Orientierung in der eigenen Wirklichkeit, in der die Dämonen des Nationalismus nach der Knebelung durch das Habsburgerreich erst so richtig losgelassen, die Gespenster des politischen 
Katholizismus nicht besiegt waren und dadurch erst die Fratzen der Faschismen verschiedener Provenienz auftauchen konnten.“ (Leitner 2014)

Ludwig Winders Thronfolger ist tatsächlich vieles in einem: Literatur, Geschichtsschreibung und Kontrafaktur. Das kontrafaktische Potential von Winders Roman ermöglicht es, diesen auch auf unsere Gegenwart zu beziehen. Der Grund dafür liegt vor allem darin, dass Winder sozusagen einen Herrscher im Potentialis zeigt und damit mögliche Zukünfte Europas. Ulrich Weinzierl schreibt im Nachwort zur Ausgabe von 2014: „Ludwig Winder war kein Habsburg-Nostalgiker, jedoch ein skeptischer Freund des multinationalen alten Österreich." (Weinzierl 2014: 566) Von daher reflektiert er in seinem Roman die Möglichkeiten, wie dieses multinationale Österreich hätte gerettet werden können, damit aber auch, was man in der eigenen Gegenwart tun könnte, um das sozusagen ,gute‘ Österreich in neuer Form zu reetablieren.

\section{Quellen und Literatur}

Andreas (2014): Ludwig Winder: Der Thronfolger. In: LiteraturBlog von LeserInnen für LeserInnen. https://www.literatur-blog.at/2014/08/ludwig-winder-der-thronfolger/ (12. 8. 2020).

Aristoteles (1995): Topik, übers. von Eugen Rolfes. Hamburg: Felix Meiner.

Gauß, Karl-Markus (2014): Kaum ein Funken Hoffnung. In: Die ZEIT 5, 23. 1. 2014. https://www. zeit.de/2014/05/ludwig-winder-franz-ferdinand/komplettansicht (12. 8. 2020).

Henneberg, Nicole (2014): Europa starb in Prag. In: Frankfurter Allgemeine Zeitung, 2. 5. 2014. https://www.buecher.de/shop/ungarn/der-thronfolger-ebook-epub/winder-ludwig/products_products/detail/prod_id/40357503/(12. 8. 2020).

Höhne, Steffen - Mayer, Franziska - Weinberg, Manfred (2017): Historischer Roman. In: Becher, Peter [u.a.] (hrsg.): Handbuch der deutschen Literatur Prags und der Böhmischen Länder. Stuttgart: Metzler, S. 358-367.

Horňáček, Milan - Schöning, Matthias (2017): Erster Weltkrieg. In: Becher, Peter [u.a.] (hrsg.): Handbuch der deutschen Literatur Prags und der Böhmischen Länder. Stuttgart: Metzler, S. 339-349.

Jäger, Christian (2005): Minoritäre Literatur: Das Konzept der kleinen Literatur am Beispiel prager- und sudetendeutscher Werke. Wiesbaden: Deutscher Universitätsverlag.

Krolop, Kurt (1984): Nachwort. In: Winder, Ludwig: Der Thronfolger. Ein Franz-Ferdinand-Roman. Berlin: Rütten \& Loening, S. 597-625.

Lehnardt, Andreas (2002): Qaddish. Untersuchungen zur Entstehung und Rezeption eines rabbinischen Gebetes. Tübingen: Mohr Siebeck.

Leitner, Thomas (2014): Biografie einer sehr unerfreulichen Persönlichkeit. Dem Vergessen entrissen: Ludwig Winders Franz-Ferdinand-Roman „Der Thronfolger“. In: Falter 11, 14. 3. 2014, S. 9. https://shop.falter.at/detail/9783552056732 (12. 8. 2020).

Müller, Harro (1988): Geschichte zwischen Kairos und Katastrophe. Historische Romane im 20. Jahrhundert. Frankfurt a. M.: Athenäum.

Pazi, Margarita (2001): Ein Versuch jüdischer deutsch-tschechischer Symbiose: Ludwig Winder [1989]. In: Pazi, Margarita: Staub und Sterne. Aufsätze zur deutsch-jüdischen Literatur. Hrsg. von Sigrid Bauschinger und Paul Michael Lützeler. Göttingen: Wallstein, S. 99-115. 
Politzer, Heinz (1937): Der Thronfolger. In: Prager Tagblatt, 28. 11. 1937 (Der Sonntag), S. 11.

Seibt, Gustav (2014): Ein Untergeher. Kein Werk der Nostalgie: Ludwig Winders Roman „Der Thronfolger" über Franz Ferdinand ist endlich wieder da. In: Süddeutsche Zeitung, 28. 6. 2014. https://www.sueddeutsche.de/kultur/literatur-zu-erzherzog-franz-ferdinand-ein-untergeher-1.2019712-0\#seite-2n (12. 8. 2020).

Victor, Walther (1937): Der Grund der Gründe. In: Escher Tageblatt, 27. 11. 1937. http://www. ipsl.cz/index.php?id=1432\&lg=de\&menu=e-forum\&sub=e-forum\&str=text.php (12. 8. 2020).

Weinzierl, Ulrich (2014): Nachwort. Ein Gerechter unter den Schreibern. Ludwig Winder und sein „Thronfolger“. In: Winder, Ludwig: Der Thronfolger. Ein Franz-Ferdinand-Roman. Wien: Paul Zsolnay, S. 553-572.

Wikipedia (2020): Gattung (Literatur). https://de.wikipedia.org/wiki/Gattung_(Literatur) (12. 8. 2020).

Winder, Ludwig (1984): Der Thronfolger. Ein Franz-Ferdinand-Roman. Berlin: Rütten \& Loening. Winder, Ludwig (2014): Der Thronfolger. Ein Franz-Ferdinand-Roman. Wien: Paul Zsolnay.

Winder, Ludwig (1930): Das Heim eines finsteren Menschen. In: Vossische Zeitung, 28. 10. 1930 (Unterhaltungsblatt Nr. 252, ohne Seitennummerierung).

Manfred Weinberg, Prof. Dr. / Manfred.Weinberg@ff.cuni.cz

Ústav germánských studií, Filozofická fakulta, Univerzita Karlova, Náměstí Jana Palacha 2, 11638 Praha 1, CZ 